\title{
Voxel-based Specific Regional Analysis System for Alzheimer's Disease (VSRAD) on 3-tesla Normal Database: Diagnostic Accuracy in Two Independent Cohorts with Early Alzheimer's Disease
}

\author{
Daichi Sone $^{1,2}$, Etsuko Imabayashi², Norihide Maikusa², Masayo Ogawa ${ }^{2}$, Noriko Sato ${ }^{3}$, Hiroshi \\ Matsuda ${ }^{2, *}$, Japanese-Alzheimer's Disease Neuroimaging Initiative \\ ${ }^{1}$ Department of Psychiatry, National Center of Neurology and Psychiatry, Tokyo, Japan \\ ${ }^{2}$ Integrative Brain Imaging Center, National Center of Neurology and Psychiatry, Tokyo, Japan \\ ${ }^{3}$ Department of Radiology, National Center of Neurology and Psychiatry, Tokyo, Japan
}

[Received May 9, 2017; Revised August 9, 2017; Accepted August 18, 2017]

\begin{abstract}
Voxel-based specific regional analysis system for Alzheimer's disease (VSRAD) software is widely used in clinical practice in Alzheimer's disease (AD). The existing VSRAD is based on the normal database with 1.5-tesla MRI scans (VSRAD-1.5T), and its utility for patients have undergone 3-tesla MRI is still controversial. We recruited 19 patients with early AD and 28 healthy controls who had undergone 3-tesla MRI scans at our institute (Cohort 1). We also used the 3-tesla MRI data of 30 patients with early AD and 13 healthy controls from the Japanese Alzheimer's Disease Neuroimaging Initiative (Cohort 2). We also created a new VSRAD based on 65 normal subjects' 3-tesla MRI scans (VSRAD-3T), and compared the detectability of AD between VSRAD-1.5T and VSRAD-3T, using receiver operating characteristic curve and area under the curve (AUC) analyses. As a result, there were no significant differences in the detectability of AD between VSRAD3T and VSRAD-1.5T, except for the whole white matter atrophy score, which showed significantly better AUC in VSRAD-3T than in VSRAD-1.5T in both Cohort $1(p=0.04)$ and $2(p<0.01)$. Generally, there were better diagnostic values in Cohort 2 than in Cohort 1. The optimal cutoff values varied but were generally lower than in the previously published data. In conclusion, for patients with 3-tesla MRI, the detectability of early AD by the existing VSRAD was not different from that by the new VSRAD based on 3-tesla database. We should exercise caution when using the existing VSRAD for 3-tesla white matter analyses or for setting cutoff values.
\end{abstract}

Key words: Alzheimer's disease; VSRAD; voxel-based morphometry; 3-tesla MRI

Alzheimer's disease (AD) is a common neurodegenerative dementia, and hippocampal atrophy is a key morphological change that is useful for the diagnosis of $\mathrm{AD}$ in clinical practice [1]. The voxel-based specific regional analysis system for Alzheimer's disease
(VSRAD) is a diagnosis-aiding program, which runs on Windows, for voxel-based morphometry based on statistical parametric mapping (SPM8) and diffeomorphic anatomical registration using the exponentiated lie (DARTEL) [2]. VSRAD is widely used in current clinical

*Correspondence should be addressed to: Dr. Hiroshi Matsuda, Integrative Brain Imaging Center, National Center of Neurology and Psychiatry, 4-1-1 Ogawa-Higashi, Kodaira, Tokyo 187-8551, Japan. Email: matsudah@ @cnp.go.jp

Copyright: () 2017 Sone D. This is an open-access article distributed under the terms of the Creative Commons Attribution License, which permits unrestricted use, distribution, and reproduction in any medium, provided the original author and source are credited. 
practice in the treatment of $\mathrm{AD}$ [3]. Although 3-tesla MRI machines have become more and more common recently, the existing VSRAD is based on a normal database obtained from 1.5-tesla MRI scans [2]. A previous paper has raised the potential concern that the difference in magnetic field intensity can provide misdiagnostic information [4]. On the other hand, one study reported insignificant differences in hippocampal volumetry between 1.5- and 3- tesla MRI scans [5]. Thus, the utility of the existing VSRAD software for patients with 3-tesla MRI scans is still controversial, and should be accurately established to enable better clinical practice in the treatment of $\mathrm{AD}$. In the current study, we demonstrated the diagnostic accuracy of the existing VSRAD using two independent cohorts with different 3-tesla MRI scans. In addition, we created new VSRAD software using a 3-tesla normal database from our institute, and compared the diagnostic values with those of the existing VSRAD.

\section{MATERIALS AND METHODS}

\section{Patients Cohort 1 - recruitment at our institute}

We recruited 19 patients with early AD and 28 healthy subjects at our institute. In this cohort, AD was diagnosed based on the clinical criteria for probable $\mathrm{AD}$ [11] and on the presence of an abnormal cortical accumulation of amyloid revealed by the visual assessment of ${ }^{11} \mathrm{C}-\mathrm{PIB}$ PET. We also visually confirmed that there was no abnormal accumulation of amyloid in healthy subjects. Table 1 describes their clinical demographics.

The MRI for this cohort was performed on a 3.0tesla MR system (Verio, Siemens, Erlangen, Germany), and 3D sagittal T1-weighted magnetization prepared rapid acquisition with gradient echo (MPRAGE) images were obtained.

All subjects gave written consent to participate in the study, which was approved by the Institutional Review Board at the National Center of Neurology and Psychiatry.

\section{Patients Cohort 2 - J-ADNI data}

In this cohort, we used data from the Japanese Alzheimer's Disease Neuroimaging Initiative (J-ADNI). The clinical and imaging protocol is described elsewhere [6]. Most participants in the J-ADNI underwent 1.5-tesla MRI scans; we selected only participants who underwent 3tesla MRI scans. The clinical demographics are also shown in Table 1.

Table 1. The demographics of Cohorts 1 and 2 and the normal databases for this study.

\begin{tabular}{lcccccc}
\hline & \multicolumn{2}{c}{ Cohort 1 - Our Institute } & \multicolumn{2}{c}{ Cohort 2 - J-ADNI } & \multicolumn{2}{c}{ Normal Databases } \\
\hline & $\begin{array}{c}\text { Early AD } \\
(\mathbf{N = 1 9 )}\end{array}$ & $\begin{array}{c}\text { Controls } \\
\mathbf{( N = 2 8 )}\end{array}$ & $\begin{array}{c}\text { Early AD } \\
(\mathbf{N = 3 0})\end{array}$ & $\begin{array}{c}\text { Controls } \\
(\mathbf{N = 1 3})\end{array}$ & $\begin{array}{c}\text { VSRAD-3T } \\
(\mathbf{N = 6 5})\end{array}$ & $\begin{array}{c}\text { VSRAD-1.5T * } \\
(\mathbf{N = 8 0})\end{array}$ \\
\hline Age (mean + SD) & $69.8 \pm 8.6$ & $66.9 \pm 7.9$ & $74.2 \pm 6.8$ & $68.2 \pm 6.0$ & $70.3 \pm 8.6$ & $70.4 \pm 7.8$ \\
Age (range) & $53-81$ & $54-86$ & $61-83$ & $61-80$ & $54-85$ & $54-86$ \\
Gender (M:F) & $6: 13$ & $15: 13$ & $12: 18$ & $6: 7$ & $30: 35$ & $37: 43$ \\
Global CDR (range) & $0.5-1.0$ & 0 & $0.5-1.0$ & 0 & N/A & N/A \\
MMSE (mean \pm SD) & $21.9 \pm 4.5$ & $29.3 \pm 1.0$ & $24.8 \pm 2.5$ & $29.8 \pm 0.6$ & N/A & $29.1 \pm 1.2$ \\
\hline * (Matsuda et al. AJNR Am J Neuroradiol. 2012) & & & & &
\end{tabular}

\section{3-tesla normal database for new VSRAD}

We created new VSRAD software (VSRAD-3T) using the 3-tesla MRI scans of 65 healthy subjects without any neuropsychiatric disorders at our institute. There were no overlapping participants with either Cohort 1 or Cohort 2. In addition, their 3-tesla MRI scans were performed on different machines (Philips Medical Systems Achieva, Best, the Netherlands; and Verio, Siemens, Erlangen, Germany). A comparison of the demographics between both normal databases in VSRAD-3T and the existing VSRAD (VSRAD-1.5T) database is presented in Table 1.

\section{Image processing and score calculation}

The 3D-T1 images of both Cohorts 1 and 2 were applied to the two different VSRAD programs (i.e., VSRAD-1.5T and VSRAD-3T). The image processing including SPM8 and DARTEL is the same in both VSRADs and was described elsewhere [2]. VSRAD generates the following scores: (1) a z-score of gray matter (GM) atrophy severity 
in the volume of interest (VOI) of AD ("Severity"); (2) the extent of GM atrophy in the VOI of AD ("Extent"); (3) the ratio of the extent of GM atrophy in the VOI to the whole brain ("Ratio"); (4) the maximum z-score of the severity of GM atrophy in the VOI of AD ("Maximum"); (5) the extent of GM atrophy in the whole brain ("Whole GM"); (6) the extent of atrophy in the white matter (WM) of the whole brain ("Whole WM"). A detailed explanation is also given in the same paper [2].

\section{Statistical Analyses}

Statistical calculation was performed for each cohort separately. We evaluated the diagnostic accuracy of each score generated from both VSRADs using receiver operating characteristic curve and area under the curve (AUC) comparisons [7]. We compared the AUC values from both VSRADs using MedCalc Software ver. 17.4 (https://www.medcalc.org/).

In addition, the MedCalc Software calculated provisional optimal cut-off values and the sensitivity/specificity for each VSRAD score. However, since the optimal cutoff values are originally dependent on the prior probability of disease [8], clinicians have to use VSRAD in view of their patients' prior probability of $\mathrm{AD}$.

\section{Morphological comparison between the normal databases of both VSRADs}

As supplementary analyses, we have performed voxelbased comparisons between the normal databases of both VSRADs. Both GM and WM images of the normal subjects, which had undergone same normalization (i.e. SPM8 and DARTEL), were compared by two-sample ttest model in SPM8 software. Differences meeting the following criteria were deemed significant: a height threshold of $\mathrm{p}<0.05$ (familywise error) and an extent threshold of $\mathrm{p}<0.001$ (false discovery rate).

Table 2. AUC values for differentiation of early AD from healthy controls using both VSRADs of ROC analysis

\begin{tabular}{lcccccccc}
\hline & \multicolumn{3}{c}{ Cohort 1- Our Institute } & & \multicolumn{3}{c}{ Cohort 2 - J-ADNI } \\
\cline { 2 - 3 } \cline { 7 - 8 } & VSRAD-3T & VSRAD-1.5T & p-value & & VSRAD-3T & VSRAD-1.5T & p-value \\
\hline Severity & 0.818 & 0.813 & 0.76 & & 0.933 & 0.931 & 0.76 \\
Extent & 0.855 & 0.820 & 0.24 & & 0.918 & 0.933 & 0.35 \\
Ratio & 0.847 & 0.806 & 0.22 & & 0.903 & 0.918 & 0.30 \\
Maximum & 0.814 & 0.792 & 0.44 & & 0.928 & 0.938 & 0.45 \\
Whole GM & 0.745 & 0.772 & 0.63 & & 0.862 & 0.797 & 0.12 \\
Whole WM & 0.723 & 0.564 & $0.04 *$ & & 0.865 & 0.662 & $<0.01^{*}$ \\
\hline
\end{tabular}

AUC: area under the curve, ROC: receiver operating characteristic, GM: gray matter, WM: white matter.

\section{RESULTS}

According to the AUC comparison (Table 2), there were no significant differences in the detectability of $\mathrm{AD}$ between VSRAD-3T and -1.5T, except for the Whole WM score, which showed a significantly better AUC in VSRAD-3T than in VSRAD-1.5T in both Cohort 1 $(\mathrm{p}=0.04)$ and Cohort $2(\mathrm{p}<0.01)$. The beeswarm plots and diagnostic values at the optimal cutoff are shown in Figure 1 and Figure 2. Generally, there were better diagnostic values and better AUCs in Cohort 2 than in Cohort 1.

For clinical use of VSRAD, we have provided the detailed Supplementary Data including all the diagnostic values at every cutoff criterion, which contains the combined Cohort 1 and 2 data as well as each separated data (Table S1-3).
Furthermore, we found significant GM and WM differences between 80 normal subjects with 1.5-tesla scans and 65 with 3-tesla scans (Fig. S1). The 1.5-tesla group showed significant GM increase in the bilateral medial frontal lobes, GM decrease in the bilateral thalami, and WM increase in the bilateral frontal lobes.

\section{DISCUSSION}

In the current study, we investigated the detectability of early $\mathrm{AD}$ patients using the existing VSRAD and a new VSRAD. For that, we used two independent cohorts with 3 -tesla MRI scans. To the best of our knowledge, this is the first study focusing on the difference in the magnetic field intensity for use in VSRAD. Consequently, there were no significant differences in most scores in both 
cohorts. Therefore, in typical clinical practice, there would be no need to use the new VSRAD based on the 3tesla normal database. Our results may support the previous report that revealed insignificant differences in hippocampal volumetry between 1.5- and 3- tesla MRI scans [5]. Another previous study compared tensor-based morphometry in AD between 1.5- and 3- tesla MRI and reported no significant difference in the detectability [9], which would accord with the results in the current study.

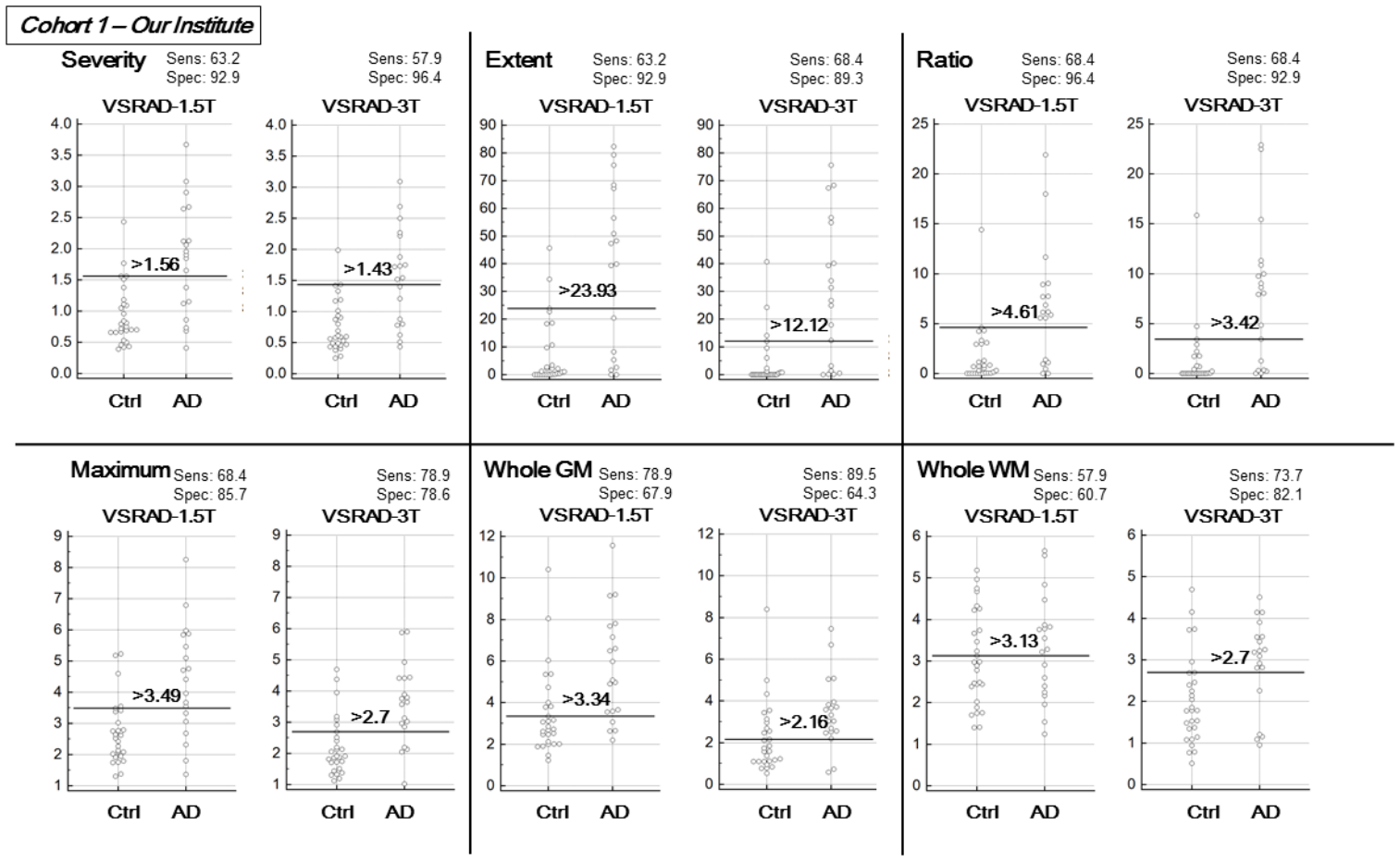

Figure 1. The beeswarm plots and diagnostic values at the optimal cutoff for each score on both VSRADs in Cohort 1.

On the other hand, in both Cohort 1 and 2, we found significantly better detectability of AD in the Whole WM score by VSRAD-3T than by VSRAD-1.5T. Although WM volume reduction would also exist in AD [10], it is unusual to use the Whole WM score on VSRAD for the diagnosis of AD in clinical practice. Therefore, this result would provide little guidance for clinical practice. However, there may exist some difference in WM volumetry between 1.5 - and 3- tesla MRI, which could be important in the field of neuroradiology. Notably, we found significant WM increase in normal subjects with 1.5-tesla scans as well as other interesting GM differences. Given the use of rigorous statistics, we consider these differences derive from the difference in magnetic field intensity. Possibly, VSRAD-1.5T may estimate less whole WM volumes and calculate high Whole WM atrophy score in normal subjects, which could lead to less AUC values for differentiation.

Another important finding in this study is the difference in the levels of detectability between the two cohorts. We found better diagnostic values and AUCs in
Cohort 2 than in Cohort 1 . The original paper on VSRAD reported that the Severity score showed high sensitivity $(86.4 \%)$ and specificity $(97.5 \%)$ for the diagnosis of very mild AD [2]. In the present study, Cohort 2 showed comparable diagnostic values, whereas less adequate results were found in Cohort 1. We speculate that the main reason for this inter-cohort difference would be aging. In Cohort 2, the patients with early AD were older than the controls by about 6 years (Table 1). This is because we enrolled all of the participants matching the criteria from the J-ADNI database without any intention or bias. The higher age in the Cohort $2 \mathrm{AD}$ patients may have enhanced the atrophy scores in both VSRADs, since the hippocampal volume shrinks with aging [11], and there is overlapping atrophy in the hippocampal body and entorhinal cortex due to both $\mathrm{AD}$ and normal aging [12]. Thus, the effect of aging must be kept in mind in order to use VSRAD accurately. Additionally, the differing detectability levels between the two cohorts may have been influenced by background factors such as differences in the MRI devices or diagnostic criteria. 


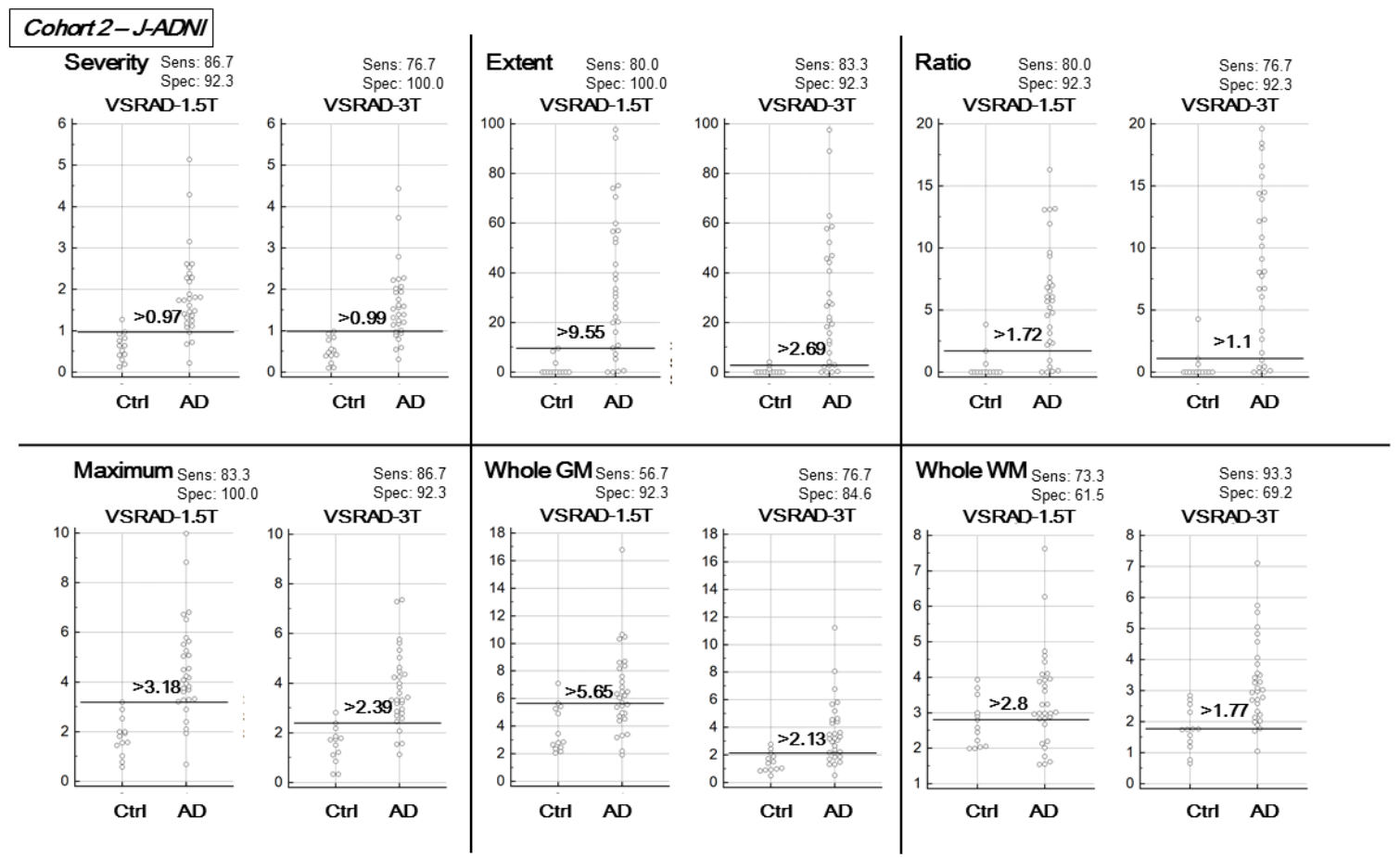

Figure 2. The beeswarm plots and diagnostic values at the optimal cutoff for each score on both VSRADs in Cohort 2.

Moreover, the cutoff values for the diagnosis of $\mathrm{AD}$ in the current study were generally lower than those in the original paper (e.g., around "2" in Severity) [2]. In particular, the optimal cutoff values for Severity were consistently around " 1 " in both cohorts. Therefore, there may exist a tendency toward lower atrophy scores in 3tesla MR images. The use of the cutoff value of "2" for the Severity score has high specificity but could lead to lower sensitivity and could cause patients potentially having early $\mathrm{AD}$ to be overlooked in 3-tesla MRI.

There are several reports of useful VSRAD applications focusing on the diagnosis of depression [13], prion disease [14], or the differentiation of $\mathrm{AD}$ and depression [15]. Some of those studies used 3-tesla MRI scans, and more studies using 3-tesla MRI and VSRAD software may emerge in the future. Our results may provide the existing VSRAD with a certain validation regarding GM analyses in 3-tesla MRI, whereas caution should be exercised in analyses of WM or in the determination of cutoff values.

This study has several limitations. First, the sample sizes in each cohort were relatively small, which might lead to the somewhat dispersed results. But we also confirmed several consistent results using the two independent cohorts. In addition, our new normal database lacked cognitive assessment (e.g., MMSE score), whereas the original VSRAD obtained such data [2]. However, we recruited only healthy subjects who reported no cognitive complaints for the normal database.
In conclusion, for patients who have undergone 3tesla MRI, the detectability of early AD using the existing VSRAD is not different from that using the new VSRAD based on a 3-tesla normal database. Caution should be exercised when using the existing VSRAD for 3-tesla WM analyses or for the setting of cutoff values.

\section{Acknowledgement}

This study was supported by the following funding: Brain Mapping by Integrated Neurotechnologies for Disease Studies (Brain/MINDS) project (grant no. 16dm 0207017h0003), funded by the Japan Agency for Medical Research and Development (AMED); and an Intramural Research Grant (27-9) for Neurological and Psychiatric Disorders from the National Center of Neurology and Psychiatry (Japan).

\section{References}

[1] Matsuda H (2016). MRI morphometry in Alzheimer's disease. Ageing Res Rev, 30: 17-24

[2] Matsuda H, Mizumura S, Nemoto K, Yamashita F, Imabayashi E, Sato N, et al. (2012). Automatic voxelbased morphometry of structural MRI by SPM8 plus diffeomorphic anatomic registration through exponentiated lie algebra improves the diagnosis of probable Alzheimer Disease. AJNR Am J Neuroradiol, 33: $1109-1114$ 
[3] Matsuda H (2013). Voxel-based Morphometry of Brain MRI in Normal Aging and Alzheimer's Disease. Aging Dis, 4: 29-37

[4] Goto M, Suzuki Y, Abe O, Hayashi N, Aoki S, Mori $\mathrm{H}$, et al. (2008). Customization of normal data base specific for 3-tesla MRI is mandatory in VSRAD analysis. Radiol Phys Technol, 1: 196-200

[5] Briellmann RS, Syngeniotis A, Jackson GD (2001). Comparison of hippocampal volumetry at 1.5 tesla and at 3 tesla. Epilepsia, 42: 1021-1024

[6] Iwatsubo T (2010). Japanese Alzheimer's Disease Neuroimaging Initiative: present status and future. Alzheimers Dement, 6: 297-299

[7] DeLong ER, DeLong DM, Clarke-Pearson DL (1988). Comparing the areas under two or more correlated receiver operating characteristic curves: a nonparametric approach. Biometrics, 44: 837-845

[8] Zweig MH, Campbell G (1993). Receiver-operating characteristic (ROC) plots: a fundamental evaluation tool in clinical medicine. Clin Chem, 39: 561-577

[9] Ho AJ, Hua X, Lee S, Leow AD, Yanovsky I, Gutman $\mathrm{B}$, et al. (2010). Comparing $3 \mathrm{~T}$ and $1.5 \mathrm{~T}$ MRI for tracking Alzheimer's disease progression with tensorbased morphometry. Hum Brain Mapp, 31: 499-514

[10] Moon CM, Shin IS, Jeong GW (2017). Alterations in white matter volume and its correlation with neuropsychological scales in patients with Alzheimer's disease: a DARTEL-based voxel-based morphometry study. Acta Radiol, 58: 204-210
Raz N, Lindenberger U, Rodrigue KM, Kennedy KM, Head D, Williamson A, et al. (2005). Regional brain changes in aging healthy adults: general trends, individual differences and modifiers. Cereb Cortex, 15: $1676-1689$

[12] Raji CA, Lopez OL, Kuller LH, Carmichael OT, Becker JT (2009). Age, Alzheimer disease, and brain structure. Neurology, 73: 1899-1905

[13] Niida R, Niida A, Motomura M, Uechi A (2011). Diagnosis of depression by MRI scans with the use of VSRAD - a promising auxiliary means of diagnosis: a report of 10 years research. Int J Gen Med, 4: 377-387

[14] Sugiyama A, Sato N, Kimura Y, Maekawa T, Wakasugi N, Sone D, et al. (2017). Thalamic involvement determined using VSRAD advance on MRI and easy Z-score analysis of 99mTc-ECDSPECT in Gerstmann-Straussler-Scheinker syndrome with P102L mutation. J Neurol Sci, 373: 27-30

[15] Shimoda K, Kimura M, Yokota M, Okubo Y (2015). Comparison of regional gray matter volume abnormalities in Alzheimers disease and late life depression with hippocampal atrophy using VSRAD analysis: a voxel-based morphometry study. Psychiatry Res, 232: 71-75. 